\title{
Planung der Behandlung
}

Sanierungsplanung. Zur Wiederherstellung der Mundgesundheit muss der Zahnarzt ein übergreifendes Gesamtkonzept erarbeiten. Kennzeichnend dafür ist eine aufeinander abgestimmte und sich ergänzende Reihenfolge von Untersuchungs- und Behandlungsmaßnahmen.

AUTOR: DR. PETER H. G. ESSER

$\mathrm{Zu}$ einem Gesamtkonzept gehören nicht nur Maßnahmen aus dem Fachgebiet der Zahnmedizin. Es stellt seinem Wesen nach keine einfache Aneinanderreihung von mehreren Heil- und Kostenplänen dar, wie gelegentlich eingewendet wird.

Aus der in bestimmten Fällen indizierten Leistung "Sanierungsplanung" resultiert eine Koordination der einzelnen Heilbehandlungsschritte. Die Planung berücksichtigt auch die nötigen zeitlichen Abfolgen wie Ausheilungs- oder Anfertigungszeiten. Damit verbunden ist eine Zuordnung der Prioritäten. Diese spezielle Planungsleistung vernetzt eine Abfolge von zahnmedizinischen und gegebenenfalls auch fachzahnärztlichen Maßnahmen mit solchen auf dem Gebiet der Medizin, Physiotherapie und/oder Verhaltenslenkung. Hinzu kommen unter Umständen Materialauswahl und auch Materialprüfung. Dazu gehört immer die Planung eines wirksamen Erhaltungskonzeptes.

\section{PRAXISBEISPIEL}

Zuerst Auswertung der Befunde und Ausarbeitung einer konservierenden Grundversorgung (0030 GOZ), danach präventive Vorbehandlung als Maßnahmengebinde. Nach Erfolgskontrollen Ausarbeitung einer PA-Behandlungsplanung (0030 GOZ) mit Planung güns- tiger Behandlungsabschnitte, eventuell medikamentöse Begleitung. Es könnte während der Parodontalbehandlung zum Beispiel eine allergologische Testung von prothetischen Materialien erfolgen. Im Anschluss daran kann die vom Erfolg der Parodontalbehandlung und den Ergebnissen der Austestung abhängige prothetische Planung (0030 bzw. $0040 \mathrm{GOZ}$ ) in Angriff genommen werden.

Eine Sanierungsplanung wird anschaulich in linearen Flussdiagrammen, meist aber in vernetzten Diagrammen visualisiert (Planungsgeflecht).

\section{WEG FÜHRT ÜBER DIE GOÄ}

Da es sich bei einer Gesamtsanierungsplanung um eine nicht in der Gebührenordnung für Zahnärzte (GOZ) beschriebene Leistung handelt und in der GOZ keine annähernd vergleichbare Leistung beschrieben ist, kann es gebührentechnisch bei derartiger Konstellation nur den Weg in die Gebührenordnung für Ärzte (GOÄ) geben: Formal käme man zu dieser Analogberechnung über den $\$ 6$ (1) und folgend $₫ 6$ (2) GOZ, der Zugänglichkeit des von Zahnärzten häufig benötigten GOÄ-Abschnittes „B. VI. Berichte, Briefe“ aufzeigt. Hier käme bewertungsmäßig und nach Art der Leistung die Ziffer Ä85 (schriftliches Gutachten je angefangene
Stunde) infrage. Falls ein Zeitaufwand von knapp zehn Minuten zahnärztlicher Zeit ausreichen sollte, wäre auch die Ziffer Ä15 „Einleitung/Koordination flankierender therapeutischer Maßnahmen" eine vom Leistungsinhalt her plausibel heranziehbare Leistung. Aus der Sanierungsplanung als übergreifendes Gesamtkonzept resultieren gegebenenfalls Überweisungen ( $\mathrm{A} 2$, Ä75) und mehrere aufeinander folgende, eigenständig berechnungsfähige Heil- und Kostenpläne. Voraussetzung sind Befundungen nach $\ddot{6} 6,1000,4000$, 8000,8080 und/oder $9000 \mathrm{GOZ}$. Einer umfassenden Sanierungsplanung geht häufig eine selbstständige, umfassende Anamneseerhebung voraus, mit systematischer Sichtung aller vorhandenen Röntgenaufnahmen und Einsichtnahme in weitere übergebene Unterlagen. Das ist keine Leistungserbringung, die mit der Ä4 (Fremdanamnese) abgehandelt ist. Diese Anamneseerhebung ähnelt der Ä30, Ä31 (homöopathische Anamnese) und könnte in Analogie dazu angesetzt und berechnet werden. Interessant ist in diesem Zusammenhang die „Honorarordnung für Zahnärzte" (HOZ) der Bundeszahnärztekammer (BZ̈̈K), die eine übergreifende Gesamtleistung "Sanierungsplanung“ zwar nicht als eine zusammenfassende Leistungsbeschreibung ausweist, jedoch über den „Heil- und Kostenplan“ hinausgehende Untersuchungs-, Simulations- und Planungsleistungen.

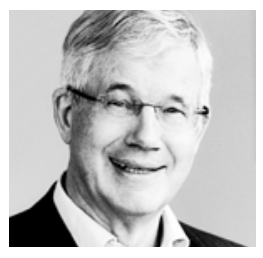

Dr. Peter H. G. Esser GOZ-Experte und Berater der ZA www.die-za.de 